


\title{
DISEÑO Y VALIDACIÓN DE UN INSTRUMENTO DE TAMIZAJE DE HABILIDADES AUDITIVAS
}

\author{
Design and Validation of a Screen \\ Instrument for Hearing Skills
}

Fecha de recepción: 5 de Junio de 2011 - Fecha de Aprobación: 15 de Agosto de 2011

\section{RESUMEN}

El procesamiento de información auditiva es un complejo sistema interrelacionado de mecanismos biológicos, fisiológicos y psicológicos, aspectos que permiten el desarrollo del lenguaje, el aprendizaje y la socialización. Este mecanismo implica habilidades tales como la detección, la discriminación, la identificación-reconocimiento y la comprensión. En el ambiente escolar se hace necesario conocer de forma rápida y eficaz el estado de estas habilidades, lo que motivó al equipo investigador el diseño y validación de un instrumento tamiz de fácil aplicación en niños de 3 a 12 años. La presente investigación de tipo descriptivo, utilizó el método de diseño y construcción de instrumentos. La evidencia de validez a partir de la evaluación de jueces expertos arrojó valores entre 0.7 y 1.0 (índice de kappa) y a partir del pilotaje de dicho instrumento se realizaron los ajustes pertinentes. Finalmente se puede concluir que dicha herramienta permite la identificación de las habilidades auditivas de la población para la que fue construida.

PALABRAS CLAVE

Procesamiento Auditivo Central, Tamizaje, Escolares, Diseño.

\begin{abstract}
Auditory information processing is a complex interrelated system of biological, physiological and psychological mechanisms, factors that enable humans to receive, transduce and understanding acoustic information from environment, aspects that enable the development of language, learning and socialization. This mechanism involves skills such as detection, discrimination, identification, recognition and understanding. In the school environment is necessary to know quickly and effectively the status of these skills, which led to the design and validation of an instrument screen is easy to apply in children 3 to 12 years. This descriptive research used the method of design and construction of instruments. The validation of content by kappa index showed a p value result between 0.7 and 1.0, construct validation through implementation of a pilot, said the need to make minor adjustments to the instrument and found that the instrument allows the identification of listening skills of the population.
\end{abstract}

KEYWORDS

Central Auditory Processing, Screening, School Design. 


\section{INTRODUCCIÓN}

$\mathrm{E}$ sentido de la audición se desarrolla desde el vientre materno permitiendo al feto reconocer sonidos internos de la madre desde la semana 25 de gestación $(1,2)$. Durante el periodo perinatal se logra el desarrollo de la corteza talámica lo que posibilita la discriminación de sonidos (3) y en la niñez se logra la madurez de la corteza cerebral $(1,2,3,4,5,6)$ lo que posibilita el desarrollo de otras habilidades auditivas que permiten la identificación el reconocimiento y la comprensión de la información recibida por este medio(7).

Acorde al desarrollo auditivo se identifican habilidades auditivas que se presentan en el trascurso del desarrollo y que son susceptibles de evaluar y promover con miras a un desarrollo integral del individuo. Las habilidades auditivas son las capacidades que tiene el individuo para captar, discriminar y comprender los estímulos sonoros. Estas habilidades se organizan en cuatro niveles secuenciales que en ocasiones se superponen. Estos son: detección, discriminación, identificación-reconocimiento y comprensión (7).

El sistema auditivo puede ser evaluado por medio de exámenes tales como la audiometría, la logoaudiometria y los reflejos estapediales, pruebas básicas que identifican dificultades en los niveles de detección y discriminación(8). Sin embargo, las habilidades auditivas van más allá de la simple detección del sonido, se hace importante identificar si el sonido detectado puede ser discriminado, reconocido, identificado y comprendido. Estas habilidades están relacionadas con el desempeño en diferentes actividades de lenguaje oral y escrito, aprendizaje y socialización $(9,10)$.

Por lo anteriormente expuesto, el presente proyecto se postula como objetivo general Diseñar y validar un instrumento tamiz de habilidades auditivas en niño entre los 3 y 12 años. Como objetivos específicos se establecen: Determinar las dimensiones de evaluación de habilidades auditivas en niños de 3 a 12 años. Determinar los indicadores de evaluación de habilidades auditivas en niños de 3 a 12 años. Determinar los ítems y criterios de evaluación de las habilidades auditivas en niños de 3 a 12 años. Hallar evidencia de validez de contenido del instrumento de evaluación de habilidades auditivas. Hallar evidencia de validez de constructo del instrumento de evaluación de habilidades auditivas.

Con el fin de comprender un poco más específicamente las dimensiones de la evaluación de la función auditiva central se describe a continuación los conceptos relacionados de detección, discriminación, identificación- reconocimiento y comprensión auditiva (7).

La Detección consiste básicamente en captar la presencia del sonido. Está habilidad permite saber que el sonido está presente o ausente. Es el primer paso para el aprendizaje auditivo y de ella dependen los niveles más altos de procesamiento.

La Discriminación consiste en poder comparar dos estímulos y determinar si son iguales o diferentes. Para esta tarea es necesario que los niños manejen dichos conceptos o que se le presenten tareas donde haya sólo dos estímulos en juego. Este nivel se plantea por las dificultades que presentan los alumnos con algunos rasgos de los sonidos del habla. Cuando se realicen este tipo de tareas hay que tener en cuenta que únicamente se están comparando dos estímulos entre sí.

La Identificación o reconocimiento se basa en la posibilidad de utilizar ciertos rasgos acústicos para seleccionar un estímulo dentro de una serie de opciones. Ya no se trata solamente de una comparación porque tiene que haber, por lo menos, más de dos estímulos presentes. La diferenciación entre Identificación y Reconocimiento se basa en cómo se realizan las tareas, ya sea en contexto cerrado (tienen los estímulos presentes o saben con certeza cuáles van a ser las opciones que se les van a presentar auditivamente) o en abierto.

En este último caso, que corresponde al Reconocimiento, se trata de una habilidad muy superior a la anterior dado que los niños no tienen referente alguno y por lo tanto tienen que valerse de su conocimiento, del contexto acústico y lingüístico y de la información almacenada en su memoria auditiva para responder.

La Comprensión, último nivel, es la habilidad que va a permitir procesar la información que se recibe por audición para construir el significado de las palabras y para decodificar los mensajes. Esta habilidad no es estrictamente auditiva ya que otras áreas de procesamiento más complejas, además de la audición, entran en juego. Pero hay que tener en cuenta que la comprensión auditiva es el mecanismo por el cual se puede utilizar la audición no sólo para la decodificación de los mensajes, sino para el desarrollo semántico, gramatical, morfológico y fonológico del lenguaje. Una vez que se haya adquirido la habilidad para comprender el lenguaje auditivamente se podrá adquirir información nueva a través de la audición sin necesidad de acceder a ella primero a través de otros sentidos, como puede ser la vista. La comprensión auditiva es un requisito para que se utilice el canal auditivo como eje central para la adquisición del lenguaje.

Uno de los elementos que más interesa en el presente estudio es "lo que hacemos con lo que escuchamos"(11), lo que el cerebro hace con las señales acústicas recibidas a través del oído. Una alteración en las habilidades de detección, discriminación, identificación y reconocimiento y comprensión auditiva pueden llegar a considerarse como inhabilidades para procesar la información auditiva lo que conlleva a la presencia de una desorden de procesamiento auditivo central. La ASHA (12) también ha definido el desorden de procesamiento auditivo central (DPAC), como "cualquier déficit en las etapas de procesamiento sensorial que comúnmente tiene impacto en la audición, en la comprensión del lenguaje hablado y el aprendizaje". The National Joint Comitee on Learning Disabilities(13) entiende el DPAC como una "dificultad en la habilidad de atender, discriminar, reconocer o comprender las informaciones presentadas auditivamente, a un individuo con audición e inteligencia normal". Estos mecanismos y procesos dependen de múltiples factores, como la indemnidad, la maduración del sistema nervioso y del sistema auditivo, además de la adecuada estimulación e interacción con el medio socioafectivo que rodea al individuo (14).

La evaluación del DPAC se realiza tradicionalmente por medio de pruebas de alto costo, requieren tiempo en su aplicación y con condiciones especiales estas pueden ser comportamentales o electrofisiológicas. Las pruebas comportamentales corresponden a test 
monocóticos, dicóticos, habla filtrada, SSW, integración biaural, pruebas de procesamiento temporal, entre otras. Uno de los procedimientos electrofisiológicos utilizados comúnmente para evaluar el procesamiento auditivo central corresponde a los potenciales evocados de tronco cerebral $(12,8)$ y la magnetoencefalografia $(15)$.

Otra forma de evaluación de las habilidades auditivas de bajo costo temporal y económico constituye en actividades comportamentales de exigencia auditiva que segmenten el uso de cierta habilidad para la realización de la misma. Algunos test de utilizados en adultos y soportados en perspectivas psicolingüísticas diseñan estrategias de evaluación de los diferentes mecanismos y habilidades de procesamiento de información auditiva $(16,17)$ e incluso en investigaciones de niños en diferentes edades se han construido instrumentos para la recolección de información sobre el procesamiento de información como el test PEHPA(15). Estas formas de evaluación resultan igualmente válidas y de gran importancia para detectar o descartar un déficit en las habilidades de procesamiento de información auditiva(18). Del mismo modo, comprender las habilidades y limitaciones dentro de los trastornos de procesamiento auditivo central contribuye a la comprensión de sus posibles abordajes $(19,20,21,22,23,24)$, considerándose requisito indispensable para la adquisición del lenguaje dentro de las bases biológicas y sociales la entrada y correcto procesamiento de la información $(24,25,26,27)$.

\section{METODOLOGÍA}

El presente estudio es de tipo descriptivo. El método que se adoptó en esta investigación es el propio de la construcción de instrumentos propuesta por Cohen \& Swerdlik (28), quienes contemplan cinco etapas: conceptualización de la prueba, construcción de la prueba, ensayo de la prueba, análisis de reactivos y revisión de la prueba.

Para la realización de la presente investigación se consultaron diferentes fuentes bibliográficas como libros, revistas y bases electrónicas, relacionadas con las siguientes temáticas: desarrollo comunicativo infantil, desarrollo auditivo, interacción comunicativa, desarrollo lingüístico, pruebas de evaluación de procesamiento auditivo central, evaluación del desarrollo auditivo (29, 30,31). De estas fuentes se identificaron las características de desarrollo comunicativo y de desarrollo auditivo de niños en edad entre los 3 y 12 años, de acuerdo a estas características se determinó las condiciones de evaluación auditiva.

Se construyó una tabla de especificaciones en la que se identificaron 4 dimensiones del constructo: Detección, Discriminación, Identificación y reconocimiento y Comprensión auditiva (ver tabla 1). Tomando como referencia estas, se construyeron 113 ítems. Estos reactivos fueron enviados a 21 jueces ( 7 jueces por grupo etario) con el fin de validar la pertinencia y la suficiencia de cada uno. Posteriormente se estructuró el instrumento acogiendo las recomendaciones hechas por los jueces y se piloteó en una muestra de 15 niños entre los 2 y los 12 años. Finalmente se realizaron nuevos cambios y ajustes tomando como referencia lo observado en el pilotaje.

\section{RESULTADOS}

Con el fin de realizar una evaluación completa de las habilidades auditivas de los niños se generó la necesidad de construir un ins- trumento que cumpliera con las condiciones de: a). permitir una evaluación rápida de las habilidades auditivas, b). utilizar la evaluación audiológica básica como fuente primordial de información sin descuidar los niveles de identificación, reconocimiento y comprensión auditiva que no se ven contemplados en esta. c). recolectar la mayor cantidad de información específica sobre el procesamiento de información auditiva a nivel central. Bajo esta premisa se identificó que el proceso de diseño de cualquier instrumento o protocolo para evaluar, debe pasar por una serie de etapas. Cohen \& Swerdlik (28), proponen que la elaboración de una prueba contempla cinco etapas: conceptualización de la prueba, construcción de la prueba, ensayo de la prueba, análisis de reactivos y revisión de la prueba.

Con las consideraciones anteriores se realizó una búsqueda de herramientas de evaluación e intervención en habilidades auditivas en niños, búsqueda que arrojó como principal herramienta el DASL II (29), los criterios de la prueba de Ling (30) y los criterios de evaluación de la prueba de lenguaje perceptivo (ESP) traducción y adaptación de la fundación CINDA(31), como fundamentos de la evaluación de habilidades auditivas, así como una serie de referencias bibliográficas de evaluación e intervención en habilidades auditivas.

Partiendo de la base investigativa ya desarrollada se realizó la selección de las dimensiones a medir: a). detección, b). discriminación, c). identificación y reconocimiento y d). comprensión auditiva. A cada una de estas dimensiones se les identificó unas subdimensiones y unos indicadores (Ver tabla 1) y luego con base en estos se elaboraron los items.

La primera habilidad referida a la detección auditiva se analiza desde las habilidades para identificar la presencia o ausencia de un estímulo sonoro tipo tono puro durante la evaluación audiológica. La segunda habilidad conocida como discriminación auditiva es susceptible de ser analizada desde la capacidad de diferenciar dos estímulos auditivos con mayores complejidades tipo fonema del habla, se identifica a partir de la diferenciación de pares mínimos en español. Implica las habilidades de diferenciar sonidos largos de cortos y graves de agudos. La tercera habilidad de Identificación y reconocimiento auditivo comprende las habilidades para reconocer que un grupo de sonidos se asocia a una imagen (contexto cerrado) o significa algo (contexto abierto). Implica las habilidades de repetir oralmente estímulos presentados de forma auditiva e identificar un referente visual. Implica las habilidades de secuenciamiento y memoria auditiva. Finalmente la comprensión auditiva, se analiza desde la capacidad de comprender oraciones y frases simples en contexto cerrado. Por medio de la exposición a ordenes simples (contexto abierto), solicitud de señalamiento de imágenes en las que se reconozca diferentes estímulos (contexto cerrado o abierto).

Para la evaluación de la habilidad de detección se decidió utilizar la prueba audiométrica como fuente de información, de modo tal que la recolección de dato fuese fiable y evitar duplicar pruebas por cada habilidad. Para la habilidad de discriminación, siendo esta una habilidad con mayor complejidad se decidió partir de los datos arrojados por la logoaudiometría, prueba que por definición busca identificar los niveles de detección de palabra y de discriminación. Sumado a este resultado se decide incluir un ítem dedicado a identi- 
3 ficar la capacidad de reconocer cada una de las características acústicas de los sonidos: tono, intensidad y duración. También se decide incluir un ítem dedicado a la identificación de pares mínimos.

\begin{tabular}{|c|c|c|}
\hline DIMENSIÓN & SUBDIMENSIONES & INDICADORES \\
\hline \multirow[b]{2}{*}{ Detección } & $\begin{array}{l}\text { Identificación de tonos } \\
\text { puros (audiometría) }\end{array}$ & $\begin{array}{l}\text { Responde a la presencia y au- } \\
\text { sencia de sonidos de diferen- } \\
\text { tes tonos }\end{array}$ \\
\hline & $\begin{array}{r}\text { Identificación de di- } \\
\text { ferencias espectrale } \\
\text { múltiples (respuesta al } \\
\text { nombre) }\end{array}$ & $\begin{array}{l}\text { Reconoce la presencia y au- } \\
\text { sencia de palabras con dife- } \\
\text { rencias espectrales múltiples } \\
\text { (nombre). }\end{array}$ \\
\hline \multirow{4}{*}{ Discriminación } & $\begin{array}{l}\text { Identificación de } \\
\text { patrones acústicos. }\end{array}$ & $\begin{array}{l}\text { Identifica la diferencia entre } \\
\text { dos sonidos por la intensidad, } \\
\text { la frecuencia (tono), la veloci- } \\
\text { dad y la duración. }\end{array}$ \\
\hline & $\begin{array}{l}\text { Identificación de } \\
\text { Pares mínimos. }\end{array}$ & $\begin{array}{l}\text { Identifica dos palabras que se } \\
\text { diferencian por un solo fonema } \\
\text { dentro de la cadena hablada y } \\
\text { señala la imagen correspon- } \\
\text { diente a la misma }\end{array}$ \\
\hline & $\begin{array}{l}\text { Identificación de Figura } \\
\text { fondo auditiva. }\end{array}$ & $\begin{array}{l}\text { Identifica la fuente sonora de } \\
\text { dos estímulos auditivos pre- } \\
\text { sentados simultáneamente }\end{array}$ \\
\hline & $\begin{array}{l}\text { Identificación de rasgos } \\
\text { acústicos } \\
\text { (logoaudiometria) }\end{array}$ & $\begin{array}{l}\text { Identifica palabras monosíla- } \\
\text { bas, bisílabas y trisílabas y las } \\
\text { señala en un dibujo }\end{array}$ \\
\hline \multirow[b]{2}{*}{$\begin{array}{l}\text { Identificación y } \\
\text { reconocimiento }\end{array}$} & $\begin{array}{l}\text { Ítems nombrados en } \\
\text { serie }\end{array}$ & $\begin{array}{l}\text { Identifica las palabras nombra- } \\
\text { das y las señala correctamente } \\
\text { guardando el orden de presen- } \\
\text { tación de los estímulos }\end{array}$ \\
\hline & Repetición de dígitos & $\begin{array}{l}\text { Identifica y repite números } \\
\text { presentados auditivamente } \\
\text { guardando el orden. Implica } \\
\text { memoria auditiva. }\end{array}$ \\
\hline \multirow{3}{*}{$\begin{array}{l}\text { Comprensión } \\
\text { auditiva }\end{array}$} & $\begin{array}{l}\text { Seguimiento de } \\
\text { instrucciones }\end{array}$ & $\begin{array}{l}\text { Comprende la instrucción y la } \\
\text { realiza fielmente en el orden } \\
\text { establecido }\end{array}$ \\
\hline & $\begin{array}{l}\text { Comprensión de un } \\
\text { párrafo }\end{array}$ & $\begin{array}{l}\text { Comprende preguntas literales } \\
\text { a partir del recuerdo de infor- } \\
\text { mación brindada tras la lectura } \\
\text { de un párrafo. }\end{array}$ \\
\hline & Ordenar una oración & $\begin{array}{l}\text { A partir de palabras aisladas y } \\
\text { presentadas oralmente cons- } \\
\text { truir una oración coherente y } \\
\text { cohesiva sin eliminar o modifi- } \\
\text { car elementos. }\end{array}$ \\
\hline
\end{tabular}

Tabla 1. Dimensiones e indicadores del constructo a medir

Es evidente que una de las habilidades que genera mayor complejidad a la hora de decodificar un mensaje oral es la discriminación de los rasgos acústicos de cada uno de los fonemas que componente la lengua al igual que segmentar ese sonido del resto de la cadena hablado, la habilidad para diferenciar dos palabras que son iguales excepto en un sonido (fonema) configura una habilidad especifica del nivel de análisis auditivo y acústico específicamente en los niveles de decodificación acústico-fonológica(25). Por lo expuesto, se decide integrar a la prueba el ítem de discriminación de pares mínimos.
Las palabras seleccionadas para conformar estos pares no fueron seleccionadas al azar. Se establecieron como criterios la presencia de fonemas con diferencias en rasgos articulatorios de modo tal que se diferencien en un solo rasgo articulatorio y con vocales que dieran cuenta de los rangos acústicos de la lengua. Así que en definitiva se seleccionan 6 pares de palabras con las los rasgos articulatorios y acústicos que se presentan en la tabla 2.

\begin{tabular}{|c|c|c|}
\hline PAR MÍNIMO & RASGO ARTICULATORIO & RASGO ACÚSTICO \\
\hline Carro- Tarro & $\begin{array}{l}\text { Cambio de punto de articu- } \\
\text { lación conservando sonori- } \\
\text { dad y modo de articulación. } \\
\text { Primera posición de pala- } \\
\text { bra. }\end{array}$ & $\begin{array}{l}\text { Vocal /a/ asociada a la con- } \\
\text { sonante cambiante. Sonido } \\
\text { Medio entre los } 600 \text { y } 1200 \\
\mathrm{~Hz} .\end{array}$ \\
\hline Cama - Casa & $\begin{array}{l}\text { Cambio de modo, sonori- } \\
\text { dad y punto de articulación. } \\
\text { Segunda posición de pala- } \\
\text { bra }\end{array}$ & $\begin{array}{l}\text { Vocal /a/ asociada a la con- } \\
\text { sonante cambiante. Sonido } \\
\text { Medio entre los } 600 \text { y } 1200 \\
\mathrm{~Hz} .\end{array}$ \\
\hline Pata-Bata & $\begin{array}{l}\text { Cambio de sonoridad entre } \\
\text { las consonantes, se con- } \\
\text { serva punto y modo de ar- } \\
\text { ticulación. Primera posición } \\
\text { de palabra. }\end{array}$ & $\begin{array}{l}\text { Vocal /a/ asociada a la con- } \\
\text { sonante cambiante. Sonido } \\
\text { Medio entre los } 600 \text { y } 1200 \\
\text { Hz. }\end{array}$ \\
\hline Piña- Niña & $\begin{array}{l}\text { Cambio de modo, sonori- } \\
\text { dad y punto de articulación, } \\
\text { incluye cambio de resona- } \\
\text { dor (oral- nasal). Primera } \\
\text { posición de palabra. }\end{array}$ & $\begin{array}{l}\text { Vocal /i/ asociada a la con- } \\
\text { sonante cambiante. Soni- } \\
\text { do agudo. Primer formante } \\
250 \mathrm{~Hz} \text { y segundo formante } \\
2500 \mathrm{HZ} \text {. }\end{array}$ \\
\hline Luna -Cuna & $\begin{array}{l}\text { Cambio de modo, sonori- } \\
\text { dad y punto de articulación, } \\
\text { incluye sonido líquido. Pri- } \\
\text { mera posición de palabra. }\end{array}$ & $\begin{array}{l}\text { Vocal /u/ asociada a la con- } \\
\text { sonante cambiante. Soni- } \\
\text { do grave. Primer formante } \\
250 \mathrm{~Hz} \text { y segundo formante } \\
500 \mathrm{HZ} \text {. }\end{array}$ \\
\hline Oreja-Oveja & $\begin{array}{l}\text { Cambio de modo y punto } \\
\text { de articulación, se mantie- } \\
\text { ne el rasgo de sonoridad. } \\
\text { Incluye cambio en la dura- } \\
\text { ción de estímulo respecto } \\
\text { a los anteriores. Segunda } \\
\text { posición de palabra. }\end{array}$ & $\begin{array}{l}\text { Vocal /e/ asociada a la con- } \\
\text { sonante cambiante. Soni- } \\
\text { do medio. Primer formante } \\
600 \mathrm{~Hz} \text { y segundo formante } \\
2000 \mathrm{HZ} \text {. }\end{array}$ \\
\hline
\end{tabular}

Tabla 2. Condiciones de los pares mínimos

Se incluyó un apartado para evaluar la figura fondo auditiva. Esta habilidad parte del reconocimiento de la fuente sonora aun cuando se presenten dos estímulos auditivos simultáneamente; identificar ambos estímulos representa hacer una identificación de rasgos acústicos de cada sonido, segmentar la información y atribuirle una fuente de sonido que además debe ser conocida y encontrarse en memoria para poder ser identificada. Por eso se seleccionan sonidos familiares para un niño de 8 años como son: Timbre - Llanto, Ladrido de un Perro - Sonarse, relinche de un Caballo - Campana. Estos sonidos además de presentarse simultáneamente cumplen con la condición de que uno se presenta de forma continua como un ruido de fondo y el otro se superpone en un momento dado, adicionalmente uno de los sonidos es más continuo y el otro es más discontinuo. Finalmente, se decide que la selección de la fuente de sonido debe 
hacerse por señalamiento dentro de un contexto cerrado de respuestas, para ello se coloca una imagen de apoyo con cuatro imágenes y el niño debe seleccionar dos de las cuales corresponden a las fuentes de sonido escuchadas.

Para la habilidad de identificación y reconocimiento auditivo las actividades tienden a complejizarse. En este nivel entra en juego no sólo el reconocimiento de patrones acústicos, la selección de uno o dos rasgos de los mismos y su asociación a un fonema, sino que entra en juego la síntesis auditiva en la cual debe identificar todos y cada uno de los elementos de los fonemas presentes en las palabras, los rasgos de duración, acentuación e intensidad de las mismas y asociarlo a una palabra, repetir la palabra o asignarle significado para evocar la imagen visual del objeto representado y poder asociarlo a una imagen. En este nivel se identifican dos niveles uno más automático de repetición aunque no exista comprensión de la palabra y uno más elaborado de asociación palabra imagen, en ambos casos se requiere del uso de la memoria de trabajo y memoria inmediata como herramientas de trabajo. En el primer nivel se decide realizar la evaluación con dígitos, dado que los dígitos pueden o no tener una condición de reconocimiento verdadera para niños de edades tempranas por no conocer su significado simbólico en estas edades. Se seleccionan aleatoriamente dígitos para solicitar al niño los repita en el orden dado. De esta manera, se evalúa la identificación de los patrones auditivos de los diferentes fonemas y combinaciones que se presentan al pronunciar los nombres. Finalmente, se seleccionan palabras de diferentes características acústicas, fáciles de representar y conocidas por los niños para que sean identificadas y reconocidas en asociación a una imagen. Las palabras se presentan en orden aleatorio y en número creciente $(2,3,4$, palabras) con el fin de identificar las habilidades de retención auditiva, memoria de trabajo y la capacidad de identificación de las distintas características auditivas de las palabras.

Finalmente, la última habilidad es la comprensión auditiva. Esta habilidad es evaluada en niños pequeños a través de la comprensión de órdenes simples y complejas. De acuerdo con las escalas de desarrollo infantil un niño a los 8 años es capaz de seguir órdenes de complejidad creciente y retener en memoria la información para ejecutarla. De acuerdo a esto se seleccionan tareas simples pero que implican la capacidad de retención de la información y la disociación de dos o tres actividades de diversa índole. Es decir, se seleccionan instrucciones que no tengan una relación de continuidad y que impliquen elementos adicionales como el uso de instrumentos (campana, llaves, etc). Así, tareas como señálate la nariz y has sonar la campana no tienen relación directa y son dos acciones separadas que no dependen la una de la otra.

Una vez identificados estas condiciones de evaluación de cada dimensión y los indicadores de su funcionamiento se procedió a identificar el nivel de dificultad para las edades comprendidas entre los 3 y 4 años, 5 y 7 años y 8 y 12 años. Para cada edad se construyó una versión del instrumento de acuerdo a criterios de niveles de dificultad acercándose al nivel de desarrollo auditivo y de lenguaje para cada edad $(7,25,26)$. La construcción del instrumento implicó la identificación de material lingüístico, visual y auditivo de aplicación confiable para las edades objeto de evaluación. Para este proceso se utilizó como referente anteriores materiales construidos y validados para población infantil, especialmente material de logoaudiometría $(32,33)$.

Luego de contar con el material lingüístico, visual y auditivo establecido se procedió a la construcción del mıınual de aplicación y ficha de registro del instrumento. El manual y la ficha pasaron por varias etapas hasta consolidarse en un instruct vo claro y breve de la aplicación. En primer lugar se identifica la prueba y se establece las edades objetivo de la misma. En segundo lugar se presenta paulatinamente cada una de las habilidades auditivas discriminando los indicadores a evaluar. En cada indicador se establece una introducción al evaluador sobre cómo aplicar el ítem, luego se presenta la instrucción que se debe dar al niño en el momento mismo de la aplicación. Posteriormente se presenta en forma de casilla para facilitar la calificación y el seguimiento de la prueba las palabras o acciones claves y un espacio para calificar. Al f.nal de cada inclicador se presenta la forma de calificación y se procede a identificar el siguienté indicador o dimensión a evaluar.

No existe una puntuación general de la prueba pues esta pretende la cualificación de las habilidades de cada niño más que la cuantificación de las respuestas obtenidas a lo largo del instrumento. Una vez aplicada la prueba el formato debe ser identificado con el nombre del niño evaluado, el nombre del evalıador y la fecha de la evaluación.

Una vez completado el diseño del instrumınto se procedió a generar un formato de validación por jueces expertos para hallar evidencia de validez. Se seleccionaron 21 jueces $\mathrm{d} \epsilon$ reconocida trayectoria en la fonoaudiología y en el trabajo con niños y que en algunos de los casos contaban con conocimientos de audiología y desarrollo auditivo. Se tomaron como criterios de evaluación la pe:tinencia y suficiencia de la instrucción de cada ítem, los estímulos lingüísticos, sonoros y visuales seleccioriados y la calific ación propruesta para cada uno. Una vez recolectada la información de realizó análisis de concordancia presentado en la tabla 3.

De acuerdo a las respuestas obtenidas se evidenció la necesidad de ajustar: los estímulos presentados en el ítem de identificación de patrones acústicos, la instrucción y los estímulos en la tarea de figura fondo auditiva, los estímulos y las forma de calificación de ítems nombrados en serie y la calificación de repetición de dígitos y seguimiento de instrucciones.

Dentro de las recomendaciones realizadas por los jueces se encontraron: seleccionar las palabras de ítems nombrados en serie por rasgos acústicos y no al azar, favorecer la intensidad y claridad de los estímulos sonoros de los dos primeros ítems de la prueba, mejorar la relación imagen sonido e imagen palabra, no solicitar la repetición de los pares mínimos únicamente el señalamiento, aumentar el nivel de complejidad de la comprensión de un párrafo.

Con base en las calificaciones obtenidas de los jueces y las recomendaciones generales se procedió a realizar los ajustes pertinentes en el instrumento. Así, que la versión final del instrumento incluyó modificaciones a los estímulos sonoros principalmente, la inclusión de dos fotos a los estímulos visuales y la modificación de algunas instrucciones para la presentación de los estímulos y la calificación 
de los ítems. El cambio más significativo consistió en la inclusión de ítems de entrenamiento previos a la presentación de la prueba.

\begin{tabular}{|c|c|c|c|}
\hline CONDICIONES EVALUADAS & & 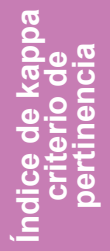 & 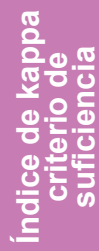 \\
\hline Discriminación & Instrucción & 0,9 & $0,6^{*}$ \\
\hline \multirow{2}{*}{$\begin{array}{l}\text { 1. Identificación de patrones } \\
\text { acústicos }\end{array}$} & Estímulos & 0,9 & $0,1^{*}$ \\
\hline & Calificación & 1,0 & 0,9 \\
\hline \multirow{3}{*}{ 2. Figura fondo auditiva } & Instrucción & 0,9 & 0,7 \\
\hline & Estímulos & 0,8 & $0,2^{*}$ \\
\hline & Calificación & 1,0 & 0,8 \\
\hline Identificación y reconocimiento & Instrucción & 1,0 & 0,9 \\
\hline \multirow{2}{*}{ 1. Pares mínimos } & Estímulos & 0,9 & $0,4^{*}$ \\
\hline & Calificación & 1,0 & 0,9 \\
\hline \multirow{3}{*}{ 2. Ítems nombrados en serie } & Instrucción & 1,0 & 0,7 \\
\hline & Estímulos & 0,9 & $0,4^{*}$ \\
\hline & Calificación & 1,0 & $0,5^{*}$ \\
\hline \multirow{3}{*}{ 3. Repetición de dígitos } & Instrucción & 1,0 & 0,7 \\
\hline & Estímulos & 0,9 & $0,5^{*}$ \\
\hline & Calificación & 1,0 & 0,7 \\
\hline Comprensión auditiva & Instrucción & 1,0 & 0,9 \\
\hline \multirow{2}{*}{ 1. Seguimiento de instrucciones } & Estímulos & 0,9 & $0,6^{*}$ \\
\hline & Calificación & 1,0 & 0,7 \\
\hline \multirow{3}{*}{ 2. Comprensión de un párrafo } & Instrucción & 1,0 & 0,9 \\
\hline & Estímulos & 1,0 & $0,4^{*}$ \\
\hline & Calificación & 1,0 & 0,8 \\
\hline \multirow{3}{*}{ 3. Organización de una oración } & Instrucción & 1,0 & 1,0 \\
\hline & Estímulos & 1,0 & 1,0 \\
\hline & Calificación & 1,0 & 1,0 \\
\hline
\end{tabular}

Tabla 3. Indice de Kappa, resultado de la validación por jueces.

Uno de los criterios de complejización consistió en seleccionar las palabras de Ítems nombrados en serie con características acústicas diversas ( ver tabla 4). En este sentido se modificaron las palabras ubicándolas de acuerdo a número de sílabas, tipo de acento y presencia de diferentes vocales y consonantes de modo que abarcaran todo el espectro acústico del español, adicionalmente se modificó el apoyo visual generando una ficha con los elementos nombrados pero en forma aislada y no en una imagen compleja como se había seleccionado en principio.

\begin{tabular}{|l|l|l|l|}
\hline & ESDRUJULA & GRAVE & AGUDA \\
\hline MONOSILABA & & & Flor \\
\hline BISILABA & & Silla & Balón \\
\hline TRISILABA & Lámpara & Señora & Pantalón \\
\hline POLISILABA & Teléfono & Escalera & Televisor \\
\hline
\end{tabular}

Tabla 4. Selección de palabras ítems nombrados en serie

Finalmente, se procedió a pilotera el instrumento ya ajustado según las recomendaciones de los jueces, participaron en esta prueba
15 niños entre los 2 y los 12 años.Los resultados de la aplicación arrojaron la necesidad de ajustar el nivel de complejidad de los ítems de repetición de dígitos, garantizar la salida de sonido de los estímulos para evitar la confusión de los mismos, eliminar la oposición graveagudo de la identificación de patrones acústicos y ajustar algunas instrucciones en todos los rangos de edad.

Luego de esta ajuste se obtuvo un producto de uso importante para la población infantil escolar.

\section{CONCLUSIÓN}

Emprender el reto de diseñar una prueba implica la capacidad de tomar decisiones sobre la marcha, poseer una definición clara sobre las condiciones metodológicas de la construcción pero sobre todo comprender el objeto de la medición. El procesamiento auditivo central es una entidad que ha venido, con el transcurrir de los años fortaleciéndose como área de trabajo y profundizándose en ella a través de la investigación. Si bien, se considera que hace parte del procesamiento de información acústico auditiva, sus límites con el procesamiento del lenguaje son difusos. Diversos autores, desde perspectivas psicolingüísticas han descrito el posible trayecto que se establece en el procesamiento de la información acústico auditiva para llegar a convertirse en el insumo de los procesos de análisis superiores y del pensamiento. La audiología como rama de estudio disciplinar ha intentado dilucidar el proceso que se entreteje anatomofisiológicamente para enlazar la información auditiva que proviene del medio ambiente y se relaciona, entrecruza neuronalmente para permitir responder a habilidades especificas del proceso de binauralidad. Habilidades que sin esta especificidad anatómica no serían posibles.

La tarea no se ha concluido, coexisten preguntas y respuestas desde varias corrientes teóricas. No es interés del presente documento entrar en debate sobre las posibles explicaciones al proceso, sino acoger una perspectiva e intentar aportar a la aplicación del mismo en los procesos de la vida cotidiana. Incluyendo aquellos que dependen de la entrada sensorial auditiva como fuente de información primaria, dentro de las que se encuentra el desarrollo del lenguaje y el aprendizaje.

El diseño de la prueba tamiz que se propone cumplió con condiciones específicas para cada uno de los ítems construidos. Características que se presentan como importantes por el nivel de aplicación y conexión entre el quehacer del especialista en audiología y la simplicidad y cotidianidad de las tareas en sí mismas. Esta propuesta no pretende ser minimalista del procesamiento auditivo central, no pretendió abarcar cada una de las habilidades que la componen, ni considerarse una herramienta única para la evaluación. Por el contrario, respeta los resultados de la evaluación audiológica básica como fuente principal de información, reconociendo su valor como pruebas "Gold" del estado auditivo de los usuarios. Proporciona algunas estrategias para analizar otros niveles de procesamiento de información, ahondar en la intrincada relación entre el procesamiento de información auditiva y la comprensión del lenguaje. Se limita a realizar una propuesta que en principio fue validada por expertos, pero que en sí requiere de procesos de confrontación con otras pruebas y con el diario vivir de la evaluación para considerarse apropiada 
a las condiciones poblacionales y a las necesidades de tamización de población escolar.

Por tanto, se considera importante continuar en la tarea de recolectar información para dilucidar cada vez más el verdadero proceso que sufre la información acústica en el proceso de percepción auditiva, relacionarla con los procesos del lenguaje y pensamiento existente, abrir nuevos caminos a comprender cómo evaluar cada habilidad de forma independiente pero sin olvidar su interrelación.
Proponer nuevas formas de evaluación y por supuesto de promoción e intervención de estas habilidades con fines de aprendizaje y desarrollo personal.

Esta propuesta más que una respuesta es una invitación al dialogo entre áreas y campos de trabajo, para llegar a profundizar cada vez más en la comprensión y aplicación de los conceptos de comunicación humana y sus desordenes, en especial en el terreno del procesamiento auditivo central.

\section{Referencia:}

\section{Las referencias a otras obras son una parte}

muy importante en la literatura científica;

\section{ya que estas permiten conocer más sobre los autores y} mantener vivas sus voces dentro del texto.

1. Zuluaga, J. (2001) Neurodesarrollo y Estimulación. Bogota, Colombia. Panamericana

2. Vergara, R. (2000). Audición y sordera. Editorial Lerner Ltda. 47-60.

3. Love Russell J., Webb Wanda G. Neurología para los especialistas del habla y del lenguaje. Editorial Médica Panamericana. Bogotá. 1989

4. Langman, J. (1964). Embriología Medica. Desarrollo Humano Normal y Anormal. Ciudad de México, México: Interamericana

5. Paparella, M. (1982). Otorrinolaringología. Buenos Aires, Argentina: Panamericana.

6. Sadler, T. W. (1993) Embriología Medica. Buenos Aires, Argentina: Panamericana.

7. Alonso, Francisco Javier. (2005).Trabajo Logopédico sobre Habilidades Auditivas. Asturias. España. pg94-127.

8. Zenker, F. y Barajas, J. Las Funciones Auditivas Centrales [en línea]. Auditio: Revista electrónica de audiología. 1 noviembre 2003. Vol. 2(2): 31-41. <http://www.auditio.com/revista/pdf/vol2/2/ 020203.pdf> [consulta: 27 mayo 2011].

9. Lasky, E. Katz, J. Central auditory processing disorders. Problems of speech, language, and learning. Univerity Park Press. Baltimroe. 1983

10. Garcia-Vazquez, Enedina, Vazquez, Luis A. y Huang, Chi-Yu. Psychological factors and language: Impact on Mexican-American students. College Student Journal, Mar1998, Vol. 32.

11. Katz, J. Handbook of clinical audiology. $4^{\circ}$ Edición. Maryland, USA. Ed. Willians and Willians. 1994

12. American Speech-Language-Hearing Association. Central auditory processing: Current status of research and implications for clinical practice. American Journal of Audiology, Vol.5 (2): 41-54. 1996
13. The National Joint Committee on Learning Disabilities. Adults With Learning Disabilities: A Call to Action. A position paper of the National Joint Committee on Learning Disabilities. The Orton Dyslexia Society. Baltimore. MD. 1985

14. Ampuero, M., Arenas, C, Cesari, F., Lange, M. y Nieto, J.(2005). Habilidades de Procesamiento Auditivo en Niños con TEL de 4 a 4 años 11 meses. Universidad de Chile.

15. Diedlera, Pietzb, Brunnerc, Hornbergerc, Bastb y Ruppa. Auditory processing in children with language-based learning problems: a magnetencephalography studyNeuroReport 2009, Vol 20 No 9.

16. Cuetos y Vega. (1998) Evaluación y Rehabilitación de las Afasias. Madrid: Ed. Medica Panamericana. 236 pg.

17. Goodglass, H. (2005). Test de Boston para el diagnóstico de la afasia. Madrid. Ed. Médica Panamericana. 3 ed.

18. Cañete, O. 2006. Desorden del procesamiento auditivo central (DPAC). Rev. Otorrinolaringol. Cir. Cabeza Cuello 2006; 66: 263-273

19. McArthur, 2009. Auditory processing disorders: can they be treated? Current Opinion in Neurology 2009, 22:137-143

20. Tamis-LeMonda Catherine S., Shannon Jacqueline D., Cabrera Natasha J., and Lamb Michael E. Fathers and Mothers at Play With Their 2- and 3-Year-Olds: Contributions to Language and Cognitive Development. Child Development, November/December 2004, Volume 75, Number 6, Pages $1806-1820$.

21. Cabrera Natasha, Shannon Jacqueline D. \& Tamis-LeMonda Catherine. Fathers' Influence on Their Children's Cognitive and Emotional Development: From Toddlers to Pre-K. Applied Development Science 2007, Vol. 11, No. 4, 208-213 
22. Movallali, Gita y Nemati, Shahroz. Difficulties in parenting hearingimpaired children. Audiology; 2009, Vol. 18 Issue 1/2, p1-11.

23. CEIF. Interacción Infantil. Procesamiento Auditivo: Pieza clave para la organización del lenguaje Disponible en www.kaiku.com.co/ bib/o5auditivo.doc

24. MUSIEK F. SHIN J. HARE C. Plasticity, Auditory Training, and Auditory Processing Disorders. Seminars in Hearing. 2002; 23 (4): 26375 .

25. Serra, M., Serrat, E., Solé, R., Bel, A. \& Aparici, M. (2000). La adquisición del lenguaje. España: Ariel psicología. 605 pg

26. Owens, Robert. Desarrollo del lenguaje. España: pearson educación. 2003

27. Vance, M., Rosen, Stuart \& Coleman, Mike. Assessing speech perception in young childrenand relationships with language skills. International Journal of Audiology 2009; 48:708_71
28. Cohen, R. \& Swerdlik, M. (2001). Pruebas y valuación psicológica. Introducción a las pruebas y a la medición. México D.F., México: Mc Graw Hill.

29. CINDA (2009). Desarrollo Auditivo en secuencia lógica DASL II. Bogotá: Fundación CINDA.

30. Ling, D. \& Moheno de Manrique Cristina. (2002). El maravilloso sonido de la palabra. México: Ed. Trillas. 242 pg.

31. CINDA (2009) Prueba de Percepción del Habla PPH. Adaptación al español del ESP.

32. Neira y Walteros (2010). Listado de Monosílabos Colombiano. Revista Colombiana de Rehabilitación. Vol 9 Pg. 20

33. Leal y Quevedo. 2011. Adaptación del Li-SRT a población con EMOC. Revista Areté. Bogotá. Vol 11. N 1. De 2011 